\title{
THE ORDERED DISTRIBUTE CONSTRAINT
}

\author{
THIERRY PETIT \\ Mines-Nantes, LINA UMR CNRS 6241 \\ 4, rue Alfred Kastler, 44307 Nantes, France. \\ Thierry.Petit@mines-nantes.fr \\ JEAN-CHARLES RÉGIN \\ Université de Nice-Sophia Antipolis, I3S, CNRS \\ 930, route des colles, BP 14506903 Sophia Antipolis cedex, France. \\ Jean-Charles.Regin@unice.fr
}

\begin{abstract}
In this paper we introduce a new cardinality constraint: Ordered Distribute. Given a set of variables, this constraint limits for each value $\mathrm{v}$ the number of times $\mathrm{v}$ or any value greater than $\mathrm{v}$ is taken. It extends the global cardinality constraint, that constrains only the number of times a value $\mathrm{v}$ is taken by a set of variables and does not consider at the same time the occurrences of all the values greater than $\mathrm{v}$. We design an algorithm for achieving generalized arc-consistency on Ordered Distribute, with a time complexity linear in the sum of the number of variables and the number of values in the union of their domains. In addition, we give some experiments showing the advantage of this new constraint for problems where values represent levels whose overrunning has to be under control. Finally, we present three extensions of our constraint that can be particularly useful in practice.
\end{abstract}

\section{Introduction}

Constraint programming is a simple and generic paradigm, which allows to represent and solve hard problems. Problems are defined by variables that take their values into finite domains, and which are subject to constraints defining the allowed combinations of values for some subsets of variables.

Encoding problems with constraint programming often requires to define cost variables involved in an objective criteria. In this context, industrial problems generally involve some constraints on costs dedicated to the characterization of the solutions acceptable in practice. These particular constraints are independent from the objective criteria.

Representing these constraints, as well as solving efficiently the related problems, form an important issue. Recent works address this issue using global constraints. A global constraint is defined on a large number of variables, and it is associated with a filtering algorithm that removes the values which cannot satisfy the constraint. The Spread and Deviation constraints ${ }^{4,11}$ enforce the balancing of values within a set of variables. Balance is often important in assignment problems, for instance the daily assignment of newborn infant patient to nurses ${ }^{3,12}$. 
In some applications, it is required to define for some subsets of variables several levels of values, and to limit for each level the maximum number of values over this level. Existing balancing constraints such as SpREad or Deviation cannot be used because they globally limit the sum of the taken values. Furthermore, since often we manipulate values representing costs, a high value $v$ is generally at least as undesirable as any of the values which are less than $v$. In this case, classical cardinality constraints such as GCC $^{9}$ are not well-suited because they limit the number of occurrences of each value taken separately.

In order to solve this issue, we introduce OrderedDistribute, a new constraint that fills in this gap by limiting, for each value $v$, the number of occurrences of $v$ and all the values greater than $v$ within a set of variables. Then, we describe an efficient filtering algorithm establishing arc consistency associated with it.

The paper is organized as follows. Section 2 gives the background useful to understand our contribution. Some motivations of our work are presented in Section 3. Section 4 discusses the reformulation of OrderedDistribute with existing arithmetic and cardinality constraints. In section 5 , we propose a filtering algorithm establishing generalized arc-consistency in a linear time complexity. We illustrate in Section 6 the practical interest of our approach by some experiments. At last, we discuss the extension of ORDEREDDISTRIBUTE with range variables representing the cardinalities as well as the aggregation of several instances of ORderedDistribute, and we conclude.

\section{Background}

A constraint network $\mathcal{N}$ is defined as a set of $n$ variables $X=\left\{x_{1}, \ldots, x_{n}\right\}$, a set of current domains $\mathcal{D}=\left\{D\left(x_{1}\right), \ldots, D\left(x_{n}\right)\right\}$ where $D\left(x_{i}\right)$ is the finite set of possible values for variable $x_{i}$, and a set $\mathcal{C}$ of constraints between variables. An assignment of values to variables in $X$ is denoted by $A(X)$, and for each $x \in X, A(X, x)$ is the value of $x$ in $A(X) . A(X)$ is valid iff $\forall x_{i} \in X, A\left(X, x_{i}\right) \in D\left(x_{i}\right)$. A constraint $C(X)$ specifies the allowed combinations of values for a set of variables $X$, that is, it defines a subset $\mathcal{R}_{C}(\mathcal{D})$ of the Cartesian product $\Pi_{x_{i} \in X} D\left(x_{i}\right)$ of the domains of variables in $X$. A feasible assignment of $C(X)$ is an assignment which is in $\mathcal{R}_{C}(\mathcal{D})$. If $A(X)$ is a feasible assignment of $C(X)$ then we say that $A(X)$ satisfies $C(X)$.

For convenience, given a value $v$ and an assignment $A(X)$, we denote by $\#(v, A(X))$ the number of time $v$ appears in $A(X)$ and by $\#(\geq v, A(X))$ the number of values $w \geq v$ that appear in $A(X)$.

Let $C$ be a constraint over the variables $X$. A support on $C$ is an assignment which satisfies $C$. A domain $D(x)$ of $x \in X$ is arc-consistent w.r.t. $C$ iff $\forall v \in D(x), v$ belongs to a valid support on $C$. $C$ is (generalized) arc-consistent (GAC) iff $\forall x_{i} \in X$, $D\left(x_{i}\right)$ is arc-consistent w.r.t. $C$. 


\section{Motivations and Definition}

To illustrate the need of the ORDEREDDistribute constraint, we present an example of cumulative scheduling where costs (i.e., values) are used to express over-loads of capacity and where constraints related to different levels of over-loads are defined.

Scheduling problems consist in ordering some activities. In cumulative scheduling, each activity requires for its execution the availability of a certain amount of renewable resource. In constraint programming, activities are represented by variables, and cumulative problems can be encoded thanks to a dedicated constraint, Cumulative ${ }^{1}$.

Let $A$ be a set of $n$ non-preemptive activities (i.e. activities that cannot be interrupted). For each $a \in A$,

- $\operatorname{start}[a]$ is the variable representing its starting point in time.

- $d u r[a]$ is the variable representing its duration.

- res $[a]$ (height of $a$ ) is the variable representing the discrete amount of resource consumed by activity $a$.

We consider cumulative problems where each activity consumes a resource.

Definition 3.1. Given one resource with a capacity limited by capa and a set $A$ of $n$ activities, at each point in time $t$ the cumulated height $h[t]$ of the activities overlapping $t$ is $h[t]=\sum_{a \in A, \operatorname{start}[a] \leq t<\operatorname{end}[a]}$ res $[a]$. The Cumulative $(A$, capa $)$ constraint ${ }^{1}$ enforces that:

C1 : For each activity $a \in A, \operatorname{start}[a]+d u r[a]=\operatorname{end}[a]$.

$\mathrm{C} 2$ : At each point in time $t, h[t] \leq$ capa.

When the time horizon (maximum latest completion time among all activities) is fixed, the problem may have no solution if no over-loads on the resource capacity are tolerated. In ${ }^{5}$, authors introduced a relaxed version of CUMULATIvE devoted to this class of problems.

Definition 3.2. The constraint SoftCumulativeSum relaxes Cumulative by introducing:

- A time horizon th.

- A relaxed capacity of the resource denoted by relax_capa with capa $\leq$ relax_capa.

- An integer variable cost $[t]$ for each point in time ${ }^{\mathrm{a}} t<t h$.

- An integer variable $o b j$.

It enforces the following constraints:

${ }^{\text {a In }}{ }^{5}$, each variable cost $[t]$ can correspond to an interval of consecutive points in time, not only one point in time. Without loss of generality and for sake of simplicity, we use a definition where the length of intervals is 1 . 
C1 : For each activity $a \in A, \operatorname{start}[a]+\operatorname{dur}[a]=\operatorname{end}[a]$.

$\mathrm{C} 2$ : At each point in time $t, h[t] \leq$ relax_capa.

C3 : For each point in time $t, \operatorname{cost}[t]=\max (0, h[t]-$ capa $)$

$\mathrm{C} 4: o b j=\sum_{t \in\{0, \ldots, m-1\}} \operatorname{cost}[t]$

Over-loads are accepted in the SoftCumulativeSum constraint and the objective variable $(o b j)$ represents the sum of the overloads. Figure 1 contains an example of this constraint.

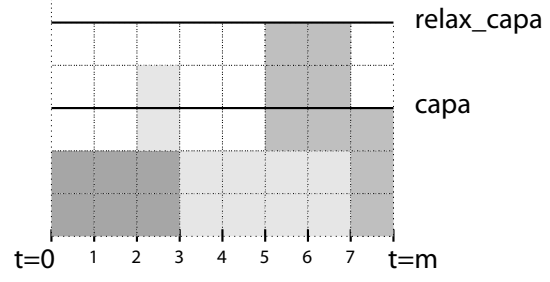

Fig. 1. Example of SoftCumulativeSum with a ground schedule with 3 fixed activities: $a_{1}$ starts at 0 and ends at 3 , and $\operatorname{res}\left[a_{1}\right]=2$. $a_{2}$ starts at 2 and ends at 7 , and res $\left[a_{2}\right]=2 . a_{3}$ starts at 5 and ends at 8 , and $\operatorname{res}\left[a_{3}\right]=3$. There is 3 over-loads: $\operatorname{cost}[2]=1, \operatorname{cost}[5]=2, \operatorname{cost}[6]=2$.

However, for some problems, constraining the sum of the over-loads is not enough. Some additional constraints w.r.t. these over-loads should be satisfied. A frequent requirement is to distribute fairly over-loads within a long time period, while limiting the number of big over-loads for each short period of time.

For instance, assume that the resource is the number of employees in a team. Each day ( 8 hours), each person contributes to some activities. An over-load will entail, in practice, either that extra-employees are hired, or that some activities are performed by a number of employees less than the number which was initially planed (for instance, some employees may accept to do homework). Typically, small overloads $(\operatorname{cost}[t]=1)$ correspond to the second case, while big ones (e.g., $\operatorname{cost}[t]>3$ ) require to hire extra-employees. Then, the number of each type of over-loads has to be limited, and these types are related one another. This means that the number of times a variable cost is greater than a given value has to be limited. Thus, we need to express that at most $k$ variables can take a value $v$ or greater.

This is the purpose of the new constraint OrderedDistribute. We define it formally:

\section{Definition 3.3. Let}

- $X$ be a set of variables

- $T$ be an array of increasing values that can be assigned to variables in $X$, with $|T| \geq 2$. 
- $I_{\max }$ be an array of maximum possible number of occurrences of values in $T$, where $I_{\max }[i]$ corresponding to the value $T[i]$, and such that $\forall i \in$ $\{1, \ldots,|T|-1\}, I_{\max }[i-1] \geq I_{\max }[i]$.

An assignment $A(X)$ satisfies the constraint OrderedDistribute $\left(X, T, I_{\max }\right)$ iff

(1) For each $i \in\{0, \ldots,|T|-1\}$, the number of values $v$ in $A(X)$ s.t. $v \geq T[i]$ is at most equal to $I_{\max }[i]$.

(2) The number of times value $T[0]$ appears in $A(X)$ is at least equals to $|X|-$ $I_{\max }[1]$.

Observe that ORDEREDDistribute also implicitly affects minimum occurrences of values: given an index $i<|T|-1$, the number of times a value $v \leq T[i]$ appears in $A(x)$ is at least equal to $|X|-I_{\max }[i+1]$.

Example 3.1. We consider a human resource cumulative problem with 5 days of 8 hours, and a team of 8 employees. The following constraints are imposed w.r.t. over-loads.

(1) At most 5 over-loaded hours by day.

(2) At most 3 over-loads greater than or equal to 2.

(3) At most 1 over-load equal to 4. (no over-load should exceed 4 employees).

Here is a fully detailed instance. We consider 40 activities with the following durations: $[1,1,3,1,4,2,2,1,4,4,2,4,2,3,1,2,1,1,1,2,1,3,3,2,4,3,3,1,4,2,4,2$, $3,4,3,4,2,3,3,2,2,2,4,4,2,4,3,4,3,4,3,2,4,4,4]$. Heights of activities are the following: $[4,2,1,1,2,3,4,1,3,3,2,1,4,4,2,3,4,1,1,1,2,4,3,3,3,1,2,4,3,2,1$, $4,2,3,4,1,4,4,1,2,1,1,4,3,2,2,1,3,4,4,3,2,4,1,1]$.

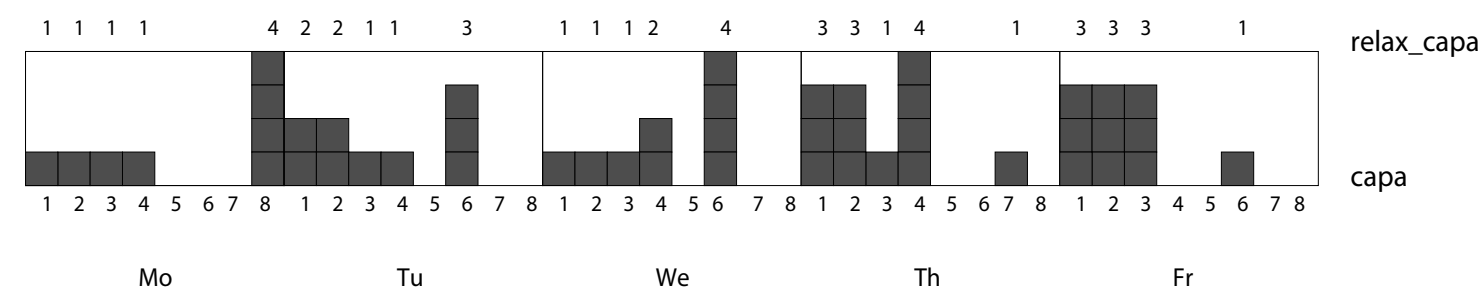

Fig. 2. Over-loads in the solution of Example 3.1.

Observe that an over-load of 4 is at least as undesirable as an over-load of 3 , an over-load of 3 is at least as undesirable as an over-load of 2 , and so on.

Figure 2 shows the over-loads in each day, in the cumulative profile corresponding to that instance. The sum of over-loads over the whole month is 48 . 
We can easily encode this problem by using the ORDEREDDistribute constraint defined with $T=[0,1,2,3,4]$ and $I_{\max }=[8,5,3,3,1]$ where $T$ measures the overloads and $I_{\max }$ limits the number of occurrences for each day. For instance, $T[2]=2$ and $I_{\max }[2]=3$ means that we can have at most 3 times an over-load of size 2 or more for each day. The following model, written in pseudo-code, represents this problem.

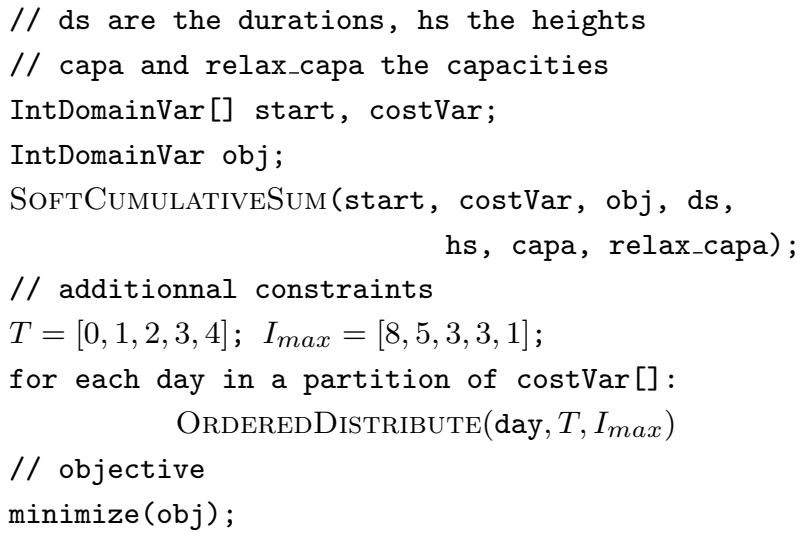

Here is a solution of the problem (each range $\left[d_{i}, e_{i}[\right.$ indicates the points in time where activity $a_{i}$ starts and ends in the schedule): $[30,31[[37,38[$ [32,35[ [12, 13[ [0,4] [24,26[ [21,23[ [15, 16[ [0,4] $[8,12[[35,37[[27,31[[26,28[[7,10[[38,39[[26,28[[31,32[$ $[39,40[[39,40[[38,40[[39,40[[12,15[[23,26[[28,30[[8,12[$ $[32,35[[32,35[[31,32[[10,14[[35,37[[32,36[[27,29[[32,35[$ $[15,19[[13,16[[32,36[[29,31[[19,22[[32,35[[36,38[[38,40[$ $[38,40[[0,4[[16,20[[36,38[[23,27[[37,40[[16,20[[20,23[$ $[4,8[[23,26[[37,39[[4,8[[32,36[[32,36[$

More generally, ORDEREDDistribute may be useful in practice in several classes of problems:

- In bin-packing problems when objects have to be packed into containers. For instance, a fair distribution based on some degrees indicating frailty of the objects allows to limits the negative consequences (in terms of financial costs) of damaged containers.

- In assignment problems when teams have to be balanced with respect to the hierarchical skills of the members.

- In over-constrained problems, in which costs represent degrees of violation of constraints. ${ }^{\mathrm{b}}$ These costs are often strongly ordered. (Example 3.1 belongs to

\footnotetext{
${ }^{\mathrm{b}} \mathrm{A}$ problem is over-constrained when it has no solution. To find compromising solutions that will
} 
this class.)

\section{Reformulation of OrderedDistribute}

Reformulating ORderedDistribute with existing cardinality constraints is not straightforward because limiting the maximum number of occurrences of a value $v$ does not constrain the occurrences of all the values which are greater than $v$. It is necessary to augment such existing cardinality constraints with additional constraints like arithmetic ones.

The most famous cardinality constraint is the global cardinality constraint or GCC. It is defined as follows:

Definition 4.1. Let $X$ be a set of variables, $T$ be an array of values, and $I$ be the array of allowed integer ranges for each value of $T$.

An assignment $A(X)$ satisfies the constraint $\operatorname{Gcc}(X, T, I)$ iff any value $v=T[i]$ appears in $A(X)$ a number of times which belongs to $I[i]$, i.e. \# $(T[i], A(X)) \in I[i]$, $i=0, \ldots,|X|-1$.

Generalized Arc Consistency (GAC, see Section 2) can be established efficiently on $\operatorname{GCC}^{9,8}$.

When $I$ are defined by boundaries of range variables (the card variables), the GCC constraint becomes the CARDVAR-GCC constraint ${ }^{10}$. A range variable is a variable which is represented by the minimum and the maximum values in its domain. Thus, the parameter $I$ is replaced by the set of range variables Card, so as the signature is CARDVAR-GCC $(X, T$, Card $)$. Filtering algorithms for CARDVAR-GCC can be found in ${ }^{10,8}$. Unfortunately, their time complexity is cubic.

Now, we can reformulate the ORDEREDDistribute constraint with a CARDVARGCC constraint and some arithmetic constraints.

Consider $C=\operatorname{OrderedDistribute}\left(X, T, I_{\max }\right)$. Let $t=|T|-1$ and $n=|X|$. We define $C N(C)$ the constraint network corresponding to the ORDEREDDistribUTE constraint as follows:

- The variable set is $X \cup \operatorname{Card}$, where $\operatorname{Card}=\{\operatorname{Card}[0], \ldots, \operatorname{Card}[t]\}$ is a set of non negative integer variables.

- The constraint set if defined by the constraints:

$$
\begin{aligned}
& -\forall k=0 \ldots t, \sum_{i=k}^{t} \operatorname{Card}[i] \leq I_{\max }[k] \\
& -\forall k=1 \ldots t, \sum_{i=0}^{k-1} \operatorname{Card}[i] \geq n-I_{\max }[k] \\
& -\operatorname{CARDVAR-GCC}(X, T, \operatorname{Card})
\end{aligned}
$$

We explicitly add the second type of constraints because as far as we know solvers are not able to deduce from two sum constraints $\sum_{i=p+1}^{n} y_{i} \leq a$ and $\sum_{i=1}^{n} y_{i}=n$ that we have $\sum_{i=1}^{p} y_{i} \geq n-a$.

be concretely applied in practice, these problems can be view as optimization problems in which some constraints may be violated. 
It is easy to check that this constraint network reformulates the constraint $C$ (i.e., they have the same set of solutions). Unfortunately, this reformulation is weak even if the strongest filtering algorithms are used for each constraint, as shown by the following example:

\section{Example 4.1.}

Let $X=\left[x_{1}, x_{2}, x_{3}, x_{4}, x_{5}\right]$ be 5 variables such that $D\left(x_{1}\right)=D\left(x_{2}\right)=\{0,1\}$, $D\left(x_{3}\right)=\{0,1,2\}$, and $D\left(x_{4}\right)=D\left(x_{5}\right)=\{2,3\}$. We consider the following constraints :

(1) At most $3 x_{i}$ greater than or equal to 1.

(2) At most $2 x_{i}$ greater than or equal to 2.

(3) At most $2 x_{i}$ equal to 3.

This example can be modeled by the constraint $C=\operatorname{OrderedDistribute}(X, T$, $\left.I_{\max }\right)$, with $T=[0,1,2,3]$ and $I_{\max }=[5,3,2,2]$. The constraint network associated with $C$ involves the constraints:

(1) $\operatorname{Card}[3] \leq I_{\max }[3]=2$

(2) $\operatorname{Card}[2]+\operatorname{Card}[3] \leq I_{\max }[2]=2$

(3) $\operatorname{Card}[1]+\operatorname{Card}[2]+\operatorname{Card}[3] \leq I_{\max }[1]=3$

(4) $\operatorname{Card}[0]+\operatorname{Card}[1]+\operatorname{Card}[2]+\operatorname{Card}[3] \leq I_{\max }[0]=5$

(5) $\operatorname{Card}[0]+\operatorname{Card}[1]+\operatorname{Card}[2] \geq|X|-I_{\max }[3]=(5-2)=3$

(6) $\operatorname{Card}[0]+\operatorname{Card}[1] \geq|X|-I_{\max }[2]=(5-2)=3$

(7) $\operatorname{Card}[0] \geq|X|-I_{\max }[1]=(5-3)=2$

(8) CARDVAR-GCC $(X, T$, Card $)$

If the strongest filtering algorithms are used for $C N(C)$ then the domains of the Card variables become $D(\operatorname{Card}[3])=\{0,1,2\} ; D(\operatorname{Card}[2])=\{0,1,2\}$; $D(\operatorname{Card}[1])=\{0,1,2,3\} ; D(\operatorname{Card}[0])=\{2,3\}$.

No value is removed from the domain of variables of $X$, whereas it should. Variables $x_{4}$ and $x_{5}$ can only take a value in $\{2,3\}$ and at the same time the number of times any value greater than or equal to 2 can be taken is 2 , because of the constraint number 2) in Example 4.1. Therefore, no other variable can take a value in $\{2,3\}$ and so value 2 can be safely removed from $D\left(x_{3}\right)$. CARDVAR-GCC does not remove such a value, because it considers, for instance, that $x_{4}$ and $x_{5}$ can take value 3 and then $x_{3}$ value 2 . In addition, note that the filtering algorithms associated with some constraints used in this reformulation are costly in practice. Thus, we have two reasons for designing an efficient filtering algorithm for the OrderedDistribute constraint. 


\section{Filtering Algorithms}

\subsection{Filtering Algorithm Based on Flow}

First, we can note that it is possible to design a filtering algorithm based on flow as for the GCC constraint. An algorithm in $O\left(n^{2} k\right)$ for checking consistency and establishing arc consistency is presented in ${ }^{6}$, where $n=|X|$ and $k=|T|$. We will not detail the algorithm here, but we will give the main idea because it solves the issue with respect to the lack of filtering of the reformulation illustrated by Example 4.1. Moreover, it shows how some arithmetic constraints between cardinality variables can be integrated into a classical filtering algorithm for GCC. This technique might also be useful to deal with generalizations of the ORDEREDDistriBute constraint.

This filtering algorithm is based on the search for a flow of value $n=|X|$ in a particular digraph. For convenience, we will denote by $l b$ the lower bound capacity of an arc and by $u b$ its upper bound capacity. Figure 3 is an example of such a digraph associated with an ORDEREDDISTRIBUTE constraint.

Definition 5.1. Let $C=\operatorname{OrderedDistribute}\left(X, T, I_{\max }\right)$, we define the digraph $G(C)=\left(X_{G}, U_{G}\right)$ as follows:

- $X_{G}=\{s, t\} \cup X \cup T$.

- $U_{G}$ contains:

- An $\operatorname{arc}(T[i], x)$ for each $T[i] \in T$ and $x \in X$ s.t. $T[i] \in D(x) . u b(T[i], x)=$ 1.

- An $\operatorname{arc}(T[i], T[i+1])$ for each $i \in\{0, \ldots,|T|-2\}$ with $u b(T[i], T[i+1])=$ $I_{\max }[i+1]$.

- An arc $(x, t)$ for each $x \in X$ with $u b(x, t)=1$.

- An $\operatorname{arc}(s, T[0])$ with $u b(s, T[0])=|X|$.

- An $\operatorname{arc}(t, s)$ with $l b(t, s)=|X|$ and $u b(t, s)=|X|$.

The main idea is to link the values together. In this way, the flow value which reaches a value $v$ of $T$ must pass by all the values less than $v$ and so it is possible to count for these values the quantity of flow corresponding to the assignments of variables to values greater than them.

Proposition 5.1. Given $C=\operatorname{OrderedDistribute}\left(X, T, I_{\text {max }}\right)$ and its corresponding digraph $G(C)$, the two following properties are equivalent:

- OrderedDistribute $\left(X, T, I_{\max }\right)$ has a solution.

- There exists a feasible flow from $t$ to $s$ in $G(C)$.

Once a feasible flow of value $n$ has been computed in $G(C)$, arc consistency can be established with the same algorithm as for GCC, so with a linear complexity.

Such a filtering technique requires to work with an additional data structure (the digraph associated with the constraint) and to compute and maintain a flow. In the next section we propose a simpler algorithm which is also more efficient in practice. 


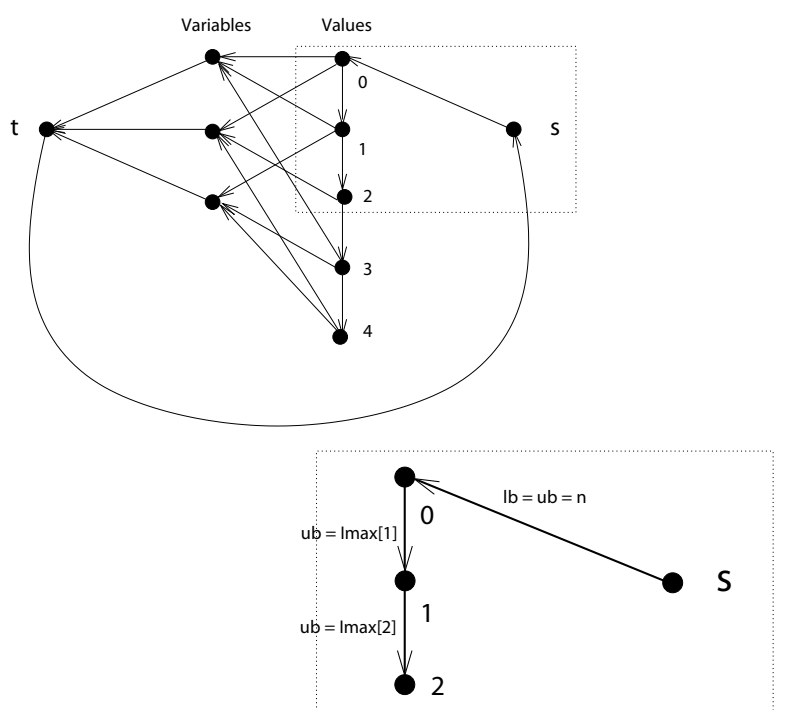

Fig. 3. Example of a digraph representing an $\operatorname{OrderedDistribute}\left(X, T, I_{\max }\right)$. Arcs between values and variables have a lower bound equal to 0 and an upper bound equal to 1 .

\subsection{Linear Filtering Algorithm}

We first come up a consistency check for ORDEREDDistribute, in a time complexity linear over the number of variables. Then, we come up with the linear GAC filtering algorithm.

By Definition 3.3, we have the following lemma.

Lemma 5.1. If OrderedDistribute $\left(X, T, I_{\text {max }}\right)$ has a solution then $I_{\text {max }}[0] \geq$ $|X|$.

We define the assignment with all minimum values of domains.

Definition 5.2. The min-domain assignment of a set of variables $X$ is the unique assignment $\underline{A}(X)$ of values to variables in $X$ s.t. $\forall x \in X, \underline{A}(X, x)$ is equal to the minimum value in $D(x)$.

Obviously, it is always possible to build a min-domain assignment if there is no empty domain, but this assignment does not necessarily satisfy the constraint.

Proposition 5.2. Let $\underline{A}(X)$ be the min-domain assignment of an instance $C$ of OrderedDistribute. The two propositions are equivalent:

$(\alpha) \#(T[0], \underline{A}(X)) \geq|X|-I_{\max }[1]$ and $\forall i=0, \ldots,|T|-1: \#(\geq T[i], \underline{A}(X)) \leq I_{\max }[i]$.

( $\beta$ ) $C$ has a solution.

Proof. $(\Rightarrow)$ Suppose that $(\alpha)$ is satisfied. By definition $3.3, \underline{A}(X)$ is a solution of $C$. $(\Leftarrow)$ Suppose that $C$ has a solution. By contradiction: assume that the min-domain 
assignment $\underline{A}(X)$ of $C$ does not satisfy $(\alpha)$. Two cases are (mutually) possible: $(i)$ The number of times $T[0]$ is assigned to a variable in $\underline{A}(X)$ is strictly less than $|X|-I_{\max }[1]$. By Definition 5.2, any variable $x$ s.t. $T[0] \in D(x)$ is assigned to $T[0]$ in $\underline{A}(X)$. Therefore, no other assignment can have a greater number of occurrences of $T[0]$ and thus satisfies $C$, a contradiction. (ii) Assume that a value $T[i]$ is s.t. $\#(\geq T[i], \underline{A}(X))>I_{\max }[i]$. By definition 5.2 , if a value greater than $T[0]$ is assigned to a variable $x$ in $\underline{A}(X)$, this value is the minimum of $D(x)$. Variables $x$ in $\underline{A}(X)$ that take value $T[i]$ cannot take a value strictly less than $T[i]$. No assignment exists with a lower value for $\#(\geq T[i], \underline{A}(X))$. $C$ has no solution, a contradiction.

From Proposition 5.2 and Definition 5.2, the feasibility of an OrderedDisTRIBUTE can be checked in $O(n)$, where $n=|X|$.

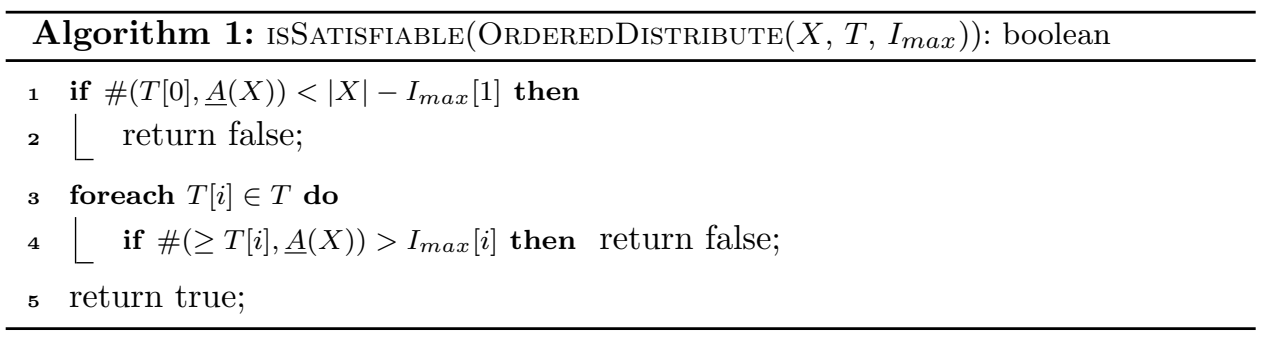

Thanks to this procedure, time complexity of a flow-based algorithm (see Section 5.1) can be decreased to $O(n k)$, where $k=|T|$. This time complexity can be improved again, by using an algorithm which does not require to work with an additionnal data structure.

We present now this dedicated filtering algorithm for ORDEREDDistribute.

Next Corollary gives a sufficient condition for having all the values consistent with OrderedDistribute.

Corollary 5.1. Let $C=\operatorname{OrderedDistribute}\left(X, T, I_{\max }\right)$, if $\forall i \in\{0, \ldots,|T|-1\}$, $\#(\geq T[i], \underline{A}(X))<I_{\max }[i]$ then $\forall x \in X, \forall v \in D(x),(x, v)$ is consistent with $C$.

Proof. The assignment $A^{\prime}(X)$ obtained by replacing $\underline{A}(X, x)$ by $v$ in $\underline{A}(X)$ is s.t. $\forall T[i] \in T, \#\left(\geq T[i], A^{\prime}(X)\right) \leq I_{\max }[i]$. By Definition 3.3, $A^{\prime}(X)$ is a solution of $C$.

Now, we can establish the corollary defining precisely the consistent values. Intuitively, there are two reasons for a value $v \in D(x)$ not to be consistent. The first one is that its variable $x$ must be assigned to $T[0]$ to satisfy the minimum requirement and $v>T[0]$. The second one is that, a maximum of occurrences is reached for a value $w \leq v$ when all variables take their minimum value and $x$ is assigned to a value $u<w$. Thus $x$ cannot be assigned to $v$ because in this case the number of value assigned to a value equal or greater than $w$ are strictly greater 
than $I_{\text {max }}[k]$ with $w=T[k]$. We denote by $X_{\perp}$ the set of variables whose domain contains $T[0]: X_{\perp}=\{x \in X$ s.t. $T[0] \in D(x)\}$.

Corollary 5.2. Let $C$ be a feasible Orderedistribute $\left(X, T, I_{\text {max }}\right)$ and $\underline{A}(X)$ be the min-domain assignment. A value $v \in D(x)$ is not consistent with $C$ if and only if one of the two following property is satisfied:

( $\alpha) x \in X_{\perp}, v>T[0]$ and $\left|X_{\perp}\right|=|X|-I_{\max }[1]$.

( $\beta) \exists T[i] \in T, \#(\geq T[i], \underline{A}(X))=I_{\max }[i]$ and $\underline{A}(X, x)<I_{\max }[i]$ and $v \geq T[i]$.

Proof. $(\alpha)$ is immediate. $(\beta)$ By definition, if in $\underline{A}(X)$ a value is assigned to a variable $x$, this value is the minimum of $D(x)$. Therefore, if $T[i]$ satisfies $\#(\geq$ $T[i], \underline{A}(X))=I_{\max }[i]$, then given $x \in X$ with $\underline{A}(X, x)<I_{\max }[i]$, there exists no assignment $A^{\prime}(X)$ s.t. $A^{\prime}(X, x) \geq I_{\max }[i]$ and $\#\left(\geq T[i], A^{\prime}(X)\right) \leq I_{\max }[i]$.

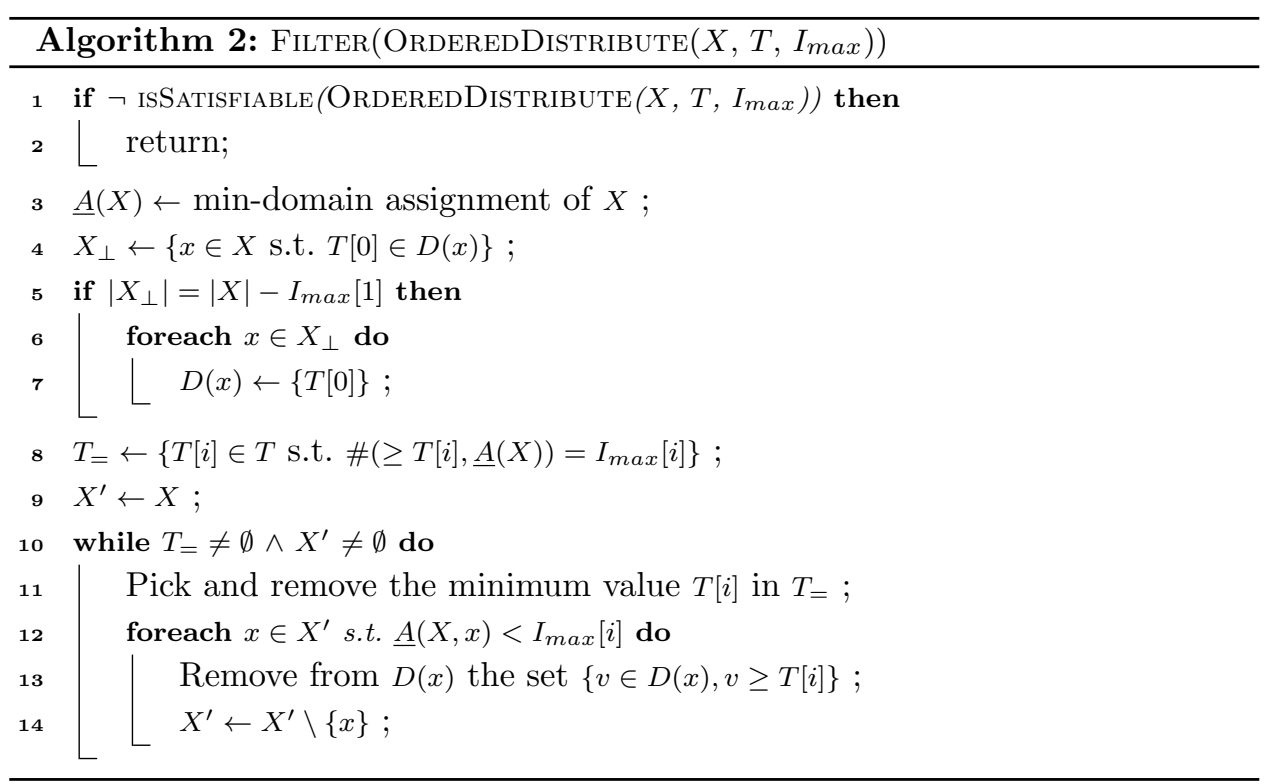

From Corollary 5.2 we obtain Algorithm 2. Values removed from a domain $D(x)$ are necessarily strictly greater than $\underline{A}(X, x)$. It is necessary to evaluate each of these values (line 10) because some new variables can be reached when evaluating higher values in $T_{=}$(defined in line 8), thanks to the condition of line 12 .

This algorithm enforces GAC. Indeed, assume that a value $T[i] \in D(x)$ is not consistent with the constraint after the run of the algorithm. This means that assigning $T[i]$ to $x$ entails in any complete assignment of $X$ the existence of a value $T[j]$ such that $\#(\geq T[j], \underline{A}(X))>I_{\max }[j]$ and $j \leq i$; especially in the mindomain assignment $\underline{A}(X)$. By construction of $T_{=}$and from line 13 of the algorithm, 
when $T[j] \in T_{=}$was considered, $T[i]$ was removed from $D(x)$ by the algorithm, a contradiction.

Proposition 5.3. The time complexity of this algorithm is $O(n+k)$, where $n=|X|$ and $k=|T|$, provided that given $v \in D(x)$, we can remove all values greater than $v$ in $O(1)$.

Proof. (Sketch) Values in $T_{=}$(line 11) and in $\underline{A}(X)$ (line 12) can be sorted in increasing order in linear time by a counting sort since $T$ is bounded. The total number of times lines $12-14$ of the algorithm are executed is upper-bounded by $|X|$ : if a variable is reached then it is removed from $X^{\prime}$ by line 14 . Thus the time complexity is in $O(|T|+|X|)$

Proposition 5.3 states that GAC can be enforced on OrderedDistribute with a time complexity linear in the sum of the number of variables and the number of values in the union of their domains. Regarding the literature, we can note that some other simple generalizations of GCC are NP-Hard ${ }^{7}$.

We can adapt Algorithm 2 to make it incremental, by maintaining at least the following data:

- The min-domain assignment $\underline{A}(X)$.

- An array $T_{\# \geq}$ counting, for each value $T[i]$, the number of values $w \geq T[i]$ that appear in $\underline{A}(X)$.

However, even in this case, each time the counter of a value in $T_{\# \geq}$ becomes such that $T_{\# \geq}[i]=I_{\max }[i]$ it will be necessary to scan the variables in order to remove all the values greater than $T_{\# \geq}[i]$ for all variables having a value in $\underline{A}(X)$ strictly less than $I_{\max }[i]$. Thus, time complexity remains in $O(|X|+|T|)$, and it can be amortized on a given branch of the search tree only w.r.t. $|T|$, which is not very relevant : Algorithm 2 has a linear time complexity and there is generally a few number of cost values. The practical time cost for updating the stored data $\underline{A}(X)$ and $T_{\# \geq}$ and the trail seems to be prohibitive. For the experiments we implemented Algorithm 2 with the version presented in this section.

\section{Experiments}

We experimented our global constraint on instances of the problem described in Example 3.1, which is derived from ${ }^{5}$, using the Java-based constraint programming engine $\mathrm{CHOCO}^{\mathrm{c}}$.

We compared two representations of OrderedDistribute.

- In the first one, we use the new global constraint we have defined. We name this model GlocalCt-Model.

${ }^{\mathrm{c}}$ http://www.emn.fr/z-info/choco-solver/index.html 
- In the second one the constraint is replaced by a constraint network equivalent to the one proposed in Section 4. We name this model CN-model.

We tried for each model several search strategies. With the first model (GlobalCtModel), the best results are obtained if, first, we first assign the minimum value to the start variable having the minimum-sized domain and then the same for cost variables. With the second model (CN-Model), the search strategy $\mathrm{dom} / w \mathrm{deg}^{2}$ was the most efficient.

Instances involve $n=55$ activities, $m=40$ hours, durations between 1 and 4 , resource consumption between 1 and 4 , capa $=8$, relax_capa $=12$. Costs at each point in time are from 0 to 4 , the imposed distribution is for each day of 8 hours : At most 5 costs $\geq 1$, at most 3 costs $\geq 2$, at most 1 cost equal to 4 .

\begin{tabular}{|c|c|c|}
\hline & $\begin{array}{c}\text { CN-Model } \\
\text { with dom/wdeg }\end{array}$ & $\begin{array}{c}\text { GlobalCt-Model } \\
\text { with StartsMinDomain }\end{array}$ \\
\hline Obj & \#Bk / Time / Proof & \#Bk / Time / Proof \\
\hline 47 & $-/>10 \min /-$ & $963 / 4 \mathrm{~s} /$ yes \\
\hline 13 & $122 / 0 \mathrm{~s} /$ yes & 50027 / 59 s / yes \\
\hline 31 & $18550 / 2 \min 52 \mathrm{~s} /$ yes & 370 / 5s / yes \\
\hline 16 & 44 / 0 s / yes & $2562 / 11 \mathrm{~s} /$ yes \\
\hline 9 & $-/>10 \min /-$ & 448192 / $10 \mathrm{~min} / \mathrm{no}$ \\
\hline 56 & $-/>10 \min /-$ & 529 / 1 s / yes \\
\hline 19 & $26 / 0 \mathrm{~s} /$ yes & 1361 / $8 \mathrm{~s} /$ yes \\
\hline 2 & 1819 / 5 s / yes & $965 / 7 \mathrm{~s} /$ yes \\
\hline 5 & 12251 / 14 s / yes & $665 / 10 \mathrm{~s} /$ yes \\
\hline 42 & $-/>10 \min /-$ & 5406 / 9s / yes \\
\hline 13 & 33484 / 38 s / yes & 4274 / 12 s / yes \\
\hline unsat. & $-/>10 \min /-$ & $-/$ > $10 \min /-$ \\
\hline 17 & $-/>10 \min /-$ & $533526 / 10 \mathrm{~min} / \mathrm{no}$ \\
\hline 48 & $-/>10 \min /-$ & $772 / 3 \mathrm{~s} /$ yes \\
\hline 33 & $-/>10 \min /-$ & $860 / 3 \mathrm{~s} /$ yes \\
\hline 56 & $-/>10 \min /-$ & $246 / 1 \mathrm{~s} /$ yes \\
\hline 46 & $-/>10 \min /-$ & 546 / $3 \mathrm{~s} /$ yes \\
\hline 14 & 54946 / 62 s / yes & 1116 / 8 s / yes \\
\hline 43 & $-/>10 \min /-$ & $615 / 3 \mathrm{~s} /$ yes \\
\hline unsat. & $-/>10 \min /-$ & $-/>10 \min /-$ \\
\hline
\end{tabular}

Table 1 gives the results obtained for instances such that no solution exists without over-loads (if there is no over-load then ORDEREDDistribute is not useful). Time limit is 10 minutes. The two unsolved problems have no solution satisfying the ordered cardinality constraints, but proving this unsatisfiability requires more 
than 10 minutes.

These results show that the use of a generic heuristic $d o m / w d e g$ which is wellsuited does not compensate the lack of filtering of the model CN-Model, except for a few number of instances which are easy to solve.

With OrderedDistribute (GlobatCt-Model), 16 of the 20 instances are solved and proved to be optimal in less than one minute ( 15 of them in less than 12 seconds), while the other model proves optimality only for 8 of the 20 instances. This latter model is not able to find a solution in a time less than 10 minutes for the 12 remaining instances. A few instances remain hard for the two models, since the optimum value cannot be found in less than 10 minutes. This is not surprising since searching for the minimum sum of over-loads in a soft cumulative problem (and proving optimality) is known to be a difficult problem.

\section{OrderedDistribute with Range Variables}

A natural extension of ORDEREDDistribute is to consider that maximum number of occurrences of values are not scalar integers but a set of range variables. We distinguish two cases.

- The first one is obtained by replacing in Definition 3.3 the integer array $I_{\max }$ by an array of range variables $R$ : the number of values greater than or equal to a given value $v$ should be less than the value of its range variable in $R$.

- The second one is similar to the first one except that the number of values greater than or equal to a given value $v$ should be equal to the value of its range variable in $R$.

\subsection{OrderedDistributeRangeLeq}

Definition 7.1. In a OrderedDistributeRangeLeq $(X, T, R)$, we use the parameters described in Definition 3.3 except that $R$ is a set of range variables. Given an assignment $A(X)$, OrderedDistribute $(X, T, R)$ is satisfied iff the two following constraints are satisfied.

(1) $\#(T[0], A(X)) \geq|X|-R[1]$.

(2) $\forall i=0, \ldots,|T|-1, \#(\geq T[i], A(X)) \leq R[i]$

The consistency check remains the same than the one of ORDEREDDistribute except that we replace $I_{\max }$ by the sequence of maximum values in domains of variables in $R$. The filtering algorithm remains the same concerning variables in $X$. It differs from OrderedDistribute w.r.t. lower bounds of domains variables in $R$ : They may become not consistent with ORDEREDDISTRIBUTERANGELEQ according to the current domains of variables in $X$. For instance, for a value $T[i]$ it may not remain enough values less than $T[i]$ in domains of variables $X$ to impose $\#(\geq T[i], \underline{A}(X)) \leq 0$, so as 0 can be removed from $R[i]$. Minimum values for variables in $R$ can be updated directly by counting, for each $T[i]$, the number of variables in $\underline{A}(X)$ greater than or 
equal to $T[i]$. This can be done incrementally while $\underline{A}(X)$ is built (note that $\underline{A}(X)$ remains the same after the filtering of variables in $X)$. At last, increasing explicitly a lower-bound of a variable $R[i]$ has no consequence except a fail if $\min (D(R[i]))>$ $\max (D(R[i]))$. Thus, GAC can be achieved on OrderedDistributeRangeLeq in $O(n+k)$ time, with $n=|X|$ and $k=|T|$.

\subsection{OrderedDistributeRangeEq}

In this section, we study the case where the number of values greater than or equal to a given value $v$ should be equal to the value of its corresponding range variable.

Definition 7.2. In a OrderedDistributeRangeEQ $(X, T, R)$, we use the parameters described in Definition 3.3 except that $R$ is a set of range variables. Given an assignment $A(X)$, OrderedDistribute $(X, T, R)$ is satisfied iff the two following constraints are satisfied.

(1) $\#(T[0], A(X)) \geq|X|-R[1]$.

(2) $\forall i=0, \ldots,|T|-1, \#(\geq T[i], A(X))=R[i]$

The filtering differs from OrderedDistributeRangeLeq. We need to compute the exact upper bounds of domains of variables in $R$ (lower bounds are given by $\underline{A}(X)$ similarly to OrderedDistributeRangeLeq). Next example shows that such a computation does not simply consists in sorting the variables $x_{i} \in X$ by non decreasing $\max \left(D\left(x_{i}\right)\right)$, then remove the first $|X|-R[1]$ ones, and then count for each $v \in T$ the number of values greater than $v$ in domains of remaining $x_{i}$ 's.

Example 7.1. Let $X=\left\{x_{1}, \ldots, x_{5}\right\}$ such that $D\left(x_{1}\right)=D\left(x_{2}\right)=\{0,4\}, D\left(x_{3}\right)=$ $\{0,3,4\}, D\left(x_{4}\right)=D\left(x_{5}\right)=\{1,2,3\} . T=[0,1,2,3,4]$. Assume that $D(R[4])=[0,1]$ and $D(R[1])=[0,|X|]$, and that we wish to prune $D(R[3])$, which is currently $[0,5]$. The maximum possible value for $R[3]$ is 4 because $x_{1}$ and $x_{2}$ cannot take both value 4 .

Consider $C=$ OrderedDistributeRangeEQ $(X, T, R)$. We search for each value $v=T[i]$ the assignment satisfying $C$ and having the maximum number of values greater than or equal to $v$. We show that it is enough to solve this problem in general. We propose to simplify the problem by only considering two values per domain of each variable.

Notation 1 . Let $v$ be a value in $T$. For any variable in $X$ let $\min (x)$ be the minimum value of the domain and $w(v, x)$ be the value of $D(x)$ which is the nearest of $v$ by excess (i.e. $w(v, x) \geq v$ and $\nexists u \in D(x)$ with $w(v, x) \geq u \geq v$ ).

Property 1 . Let $v$ be a value in $T$ and $A(X)$ be any assignment satifying $C$ with $\#(\geq v, A(X))=j$. Then, the assignment $A^{\prime}(X)$ defined from $A(X)$ as follows:

- $A^{\prime}(X, x)=\min (x)$ iff $A(X, x)<v$ 
- $A^{\prime}(X, x)=w(v, x)$ iff $A(X, x) \geq v$

satisfies the constraint $C$ and satisfies $\#\left(\geq v, A^{\prime}(X)\right)=j$.

Proof. Clearly we have $\#\left(\geq v, A^{\prime}(X)\right)=j$. In addition, since each value of $A(X)$ is replaced by a smaller value it is clear that if $A(X)$ satisfies $C$ then $A^{\prime}(X)$ also. $\square$

We propose the following greedy algorithm:

(1) We order in $L$ the variables by their non increasing min value. We break tie by non decreasing $w$ value.

(2) We repeat the following process until $L$ is empty:

We take the first variable $x$ of $L$ and remove it from $L$; and we assign $w(v, x)$ to $x$ if it does not violate $C$; otherwise we assign $\min (x)$ to $x$.

(3) The obtained assignment maximizes the number of values greater than $v$.

To prove correctness of this algorithm we introduce two lemmas. Let $A(X)$ be any assignment satisfying $C$ and two variables $x_{1}$ and $x_{2}$ :

Lemma 7.1. Assume $w\left(v, x_{1}\right)=A\left(X, x_{1}\right)$ and $\min \left(x_{2}\right)=A\left(X, x_{2}\right)$. If $\min \left(x_{1}\right) \leq$ $\min \left(x_{2}\right)$ and $w\left(v, x_{1}\right) \geq w\left(v, x_{2}\right)$ then the assignment $A^{\prime}(X)$ with $\min \left(x_{1}\right)=$ $A^{\prime}\left(X, x_{1}\right)$ and $w\left(v, x_{2}\right)=A^{\prime}\left(X, x_{2}\right)$ and $\forall y \in X-\left\{x_{1}, x_{2}\right\}: A^{\prime}(X, y)=A(X, y)$ satisfies $C$ and has the same number of values greater than or equal to $v$ as $A(X)$.

Proof. $A^{\prime}(X)$ has obviously the same number of values greater than or equal to $v$ as $A(X) . A(X)$ contains the set of value $V$ and the values $w\left(v, x_{1}\right)$ and $\min \left(x_{2}\right)$; and $A^{\prime}(X)$ contains the set of value $V$ and the values $\min \left(x_{1}\right)$ and $w\left(v, x_{2}\right)$. Since $\min \left(x_{1}\right) \leq \min \left(x_{2}\right)$ and $w\left(v, x_{2}\right) \leq w\left(v, x_{1}\right)$ then if $A(X)$ satisfies $C$ then $A^{\prime}(X)$ also.

Lemma 7.2. Assume $w\left(v, x_{1}\right)=A\left(X, x_{1}\right)$, $\min \left(x_{2}\right)=A\left(X, x_{2}\right), x_{1}$ is the variable s.t. $w\left(v, x_{1}\right)<w\left(v, x_{2}\right)$ and $\nexists y \in X-\left\{x_{1}, x_{2}\right\}$ with $w\left(v, x_{1}\right)<w(v, y) \leq w\left(v, x_{2}\right)$ and $\#\left(\geq w\left(v, x_{2}\right), A(X)\right)<R[k]$ and $T[k]=w\left(v, x_{2}\right)$. If $\min \left(x_{1}\right) \leq \min \left(x_{2}\right)$ then the assignment $A^{\prime}(X)$ with $\min \left(x_{1}\right)=A^{\prime}\left(X, x_{1}\right)$ and $w\left(v, x_{2}\right)=A^{\prime}\left(X, x_{2}\right)$ and $\forall y \in X-\left\{x_{1}, x_{2}\right\}: A^{\prime}(X, y)=A(X, y)$ satisfies $C$ and has the same number of values greater than or equal to $v$ as $A(X)$.

Proof. $A^{\prime}(X)$ has obviously the same number of values greater than or equal to $v$ as $A(X) . A(X)$ contains the set of value $V$ and the values $w\left(v, x_{1}\right)$ and $\min \left(x_{2}\right)$; and $A^{\prime}(X)$ contains the set of value $V$ and the values $\min \left(x_{1}\right)$ and $w\left(v, x_{2}\right)$. We have $w\left(v, x_{1}\right)<w\left(v, x_{2}\right)$ and $\#\left(\geq w\left(v, x_{2}\right), A(X)\right)<R[k]$ and $T[k]=w\left(v, x_{2}\right)$ hence by exchanging $w\left(v, x_{2}\right)$ and $w\left(v, x_{1}\right)$ the assignment remains a solution.

We prove by induction that it is enough to assign $w(v, x)$ to $x$ when $x$ has to be assigned and $D(x)=\{\min (x), w(v, x)\}$. It is obviously true when there is only 
one variable (if the min value is taken then the obtained assignment has less value greater than $v$ than when $w$ is taken).

Thus, consider that the current variable that has to be assigned within the greedy algorithm is $x$, with $D(x)=\{\min (x), w(v, x)\}$. Suppose that we assign $\min (x)$ to $x$. We will show that we can obtain an equivalent or "better" solution (which maximizes the number of values greater than $v$ ) by taking $w(v, x)$. After assigning $\min (x)$ to $x$, the greedy algorithm is continued and an assignment $A(X)$ is computed. This assignment satisfies the OrderedDistribute constraint. Now, if we impose $A(X, x)=w(v, x)$ and if the assignment remains a solution then the current solution is improved and it is better to assign $x$ with $w(v, x)$. Therefore, we consider that this swap between $\min (x)$ and $w(v, x)$ is not possible. Consider $Y$ the set of variables assigned after $x$. Note that, by definition of the greedy algorithm any variable $y \in Y$ satisfies $\min (y) \leq \min (x)$. Then, we have two possible cases:

- There exists a variable $y \in Y$ with $w(v, y) \geq w(v, x)$. Then, Lemma 7.1 can be applied. This means that we can safely assign $w(v, x)$ to $x$.

- Each variable $y \in Y$ satisfies $w(v, y)<w(v, x)$. We consider the one with the $w$ value which is the closest to $w(v, x)$ (that is, we define $z \in Y$ satisfying $\exists y \in Y-\{z\}$ with $w(v, z)<w(v, y)<w(v, x))$. At the moment where $x$ has been assigned, $\min (x)$ and $w(v, x)$ were in its domain, therefore we had $\#(\geq w(v, x), A p(X))<R[k]$ and $T[k]=w(v, x)$, where $A p(X)$ was a partial assignment. By the absence of variable of $Y$ with $w(v, y)=w(v, x)$ and by the definition of $z$ it means that this property still holds for $A(X)$. Therefore, Lemma 7.2 can be applied and we can safely assign $x$ to $w(v, x)$.

Thus, in all the cases it is safe to assign $x$ to $w(v, x)$ and this leads to an equivalent or better solution.

Property 2. The greedy algorithm can be applied for each value $v$ of $T$ with an overall time complexity in $O\left(n k+k^{2}\right)$.

Proof. (Sketch) The complexity for one value $v$ depends on the computation of $w(v, x)$ for each variable and the double sorts (check of consistency can be done in constant time each time a variable is fixed). Consider $n=|X|$ and $k=|T|$. Each sort can be performed in $O(k)$ by a counting sort. The computation of $w(v, x)$ can be done for each variable in $O(\log (k))$. So for one value $v$ we obtain a complexity in $O(n \log (k)+k)$. However, when running the greedy algorithm for each value $v$ we can amortize some computations: All the $w$ values for a variable $x$ can be computed in $O(k)$ by traversing the domain while $v$ is increased. Since there are $k$ values to consider, the overall complexity is $O\left(n k+k^{2}\right)$.

\section{Aggregations of OrderedDistribute}

The main usage of OrderedDistribute is depicted by Example 3.1 and Figure 2: In order to obtain a particular distribution of costs, cost variables are partitioned and 
an instance of ORDEREDDISTRIBUTE is set on each subset of variables corresponding to a class of the partition. In the Example, a day is represented by a sequence of 8 cost variables representing over-loads during the 8 hours of this day. For each day an instance of ORDEREDDistribute is set on the over-loads variables.

Additionally, an objective is usually defined over the whole set of cost variables, represented by an objective variable $o b j$ and an objective constraint. In the Example, we wish to minimize the sum of over-loads during a week of five days, that is, 40 cost variables.

We focus in this section on two classical cases for this objective constraint: either minimize the sum of costs, $o b j=\sum_{x \in X} x$, or minimize the maximum value assigned to a variable, that is, $o b j=\max _{x \in X} x$.

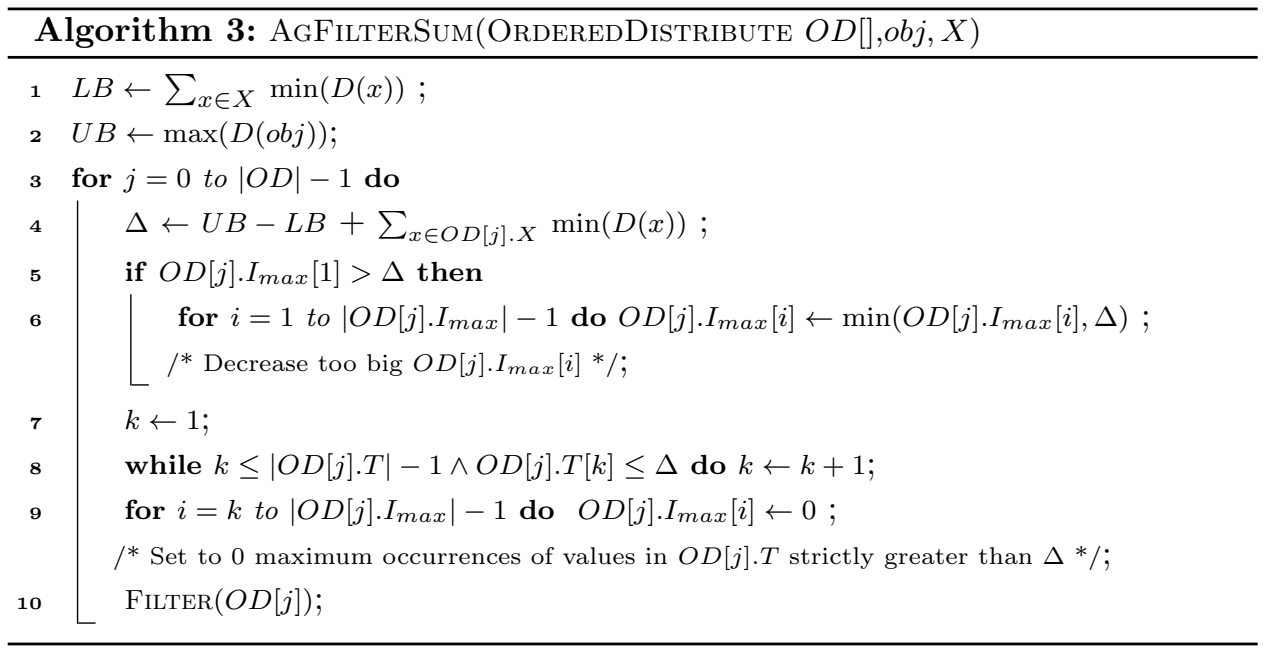

Consider a sequence $X$ of variables, partitioned in subsequences such that an instance of ORDEREDDistribute is set on each subsequence. Let us first focus on the case where the objective is a sum. The sum of minimum values in domains of variables in $X$ provide a lower bound $L B$ for the $o b j$ variable. From this lower bound, we can update arguments of each instance of ORDEREDDISTRIBUTE before calling the filtering algorithm.

- If $\max (D(o b j))$ minus $L B$ plus the sum $\sum\left(\min \left(D\left(x_{i}\right)\right)\right.$ of variables $x_{i}$ involved in the current instance of OrderedDistribute is greater than $I_{\max }[1]$, then we can safely decrease $I_{\max }[1]$ : The maximum number of values greater than or equal $T[1]$ in a solution satisfying the objective constraint (more precisely, in a solution with an objective value less than or equal to $\max (D(o b j)))$ is $\max (D(o b j))$ minus $L B$ plus the sum $\sum\left(\min \left(D\left(x_{i}\right)\right)\right.$ of variables $x_{i}$ involved in the current instance of ORderedDistribute. The same reasoning can be applied on $I_{\max }[2]$, and so on, until the condition is not satisfied (recall that, 
by Definition 3.3, values in the array $I_{\max }$ decrease as the index increases).

- Since we make no hypothesis with respect to the implementation of the objective constraint, quantity $I_{\max }[v]$ of values $v$ which cannot be assigned to any variable in $X$ without exceeding $\max (o b j)$ can be set to 0 .

Algorithm 3 implements these two principles.

The set of instances of ORDEREDDistribute are stored in an array $O D[]$ of size $|O D|$, indexed from 0 to $|O D|-1$. Arguments of one particular OrderedDisTRIBUTE at index $j$ of the $O D$ OrDEREDDISTRIBUte array are denoted respectively by $O D[j] . X, O D[j] . I_{\max }$, and $O D[j] . T$. To simplify notations, Algorithm 3 considers that each $O D[j] . I_{\max }$ is storable: values which have been modified at this node are automatically re-assigned to the previous value when a backtrack occurs.

Algorithm 4 implements the same idea for the objective constraint $o b j=$ $\max _{x \in X} x$, using the same conventions. This case is simpler since one has just to avoid exceeding the maximum value of the objective variable $o b j$.

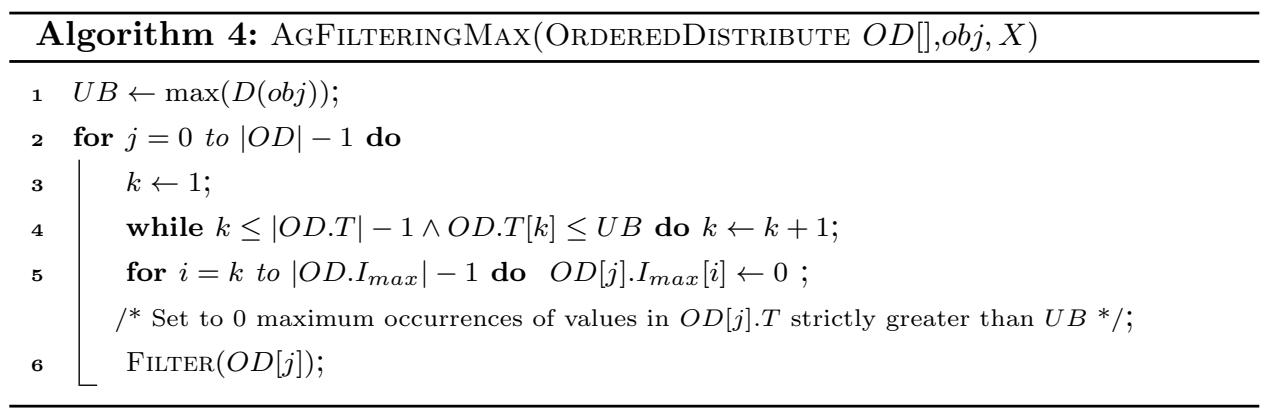

Note that Algorithm 3 and Algorithm 4 can work with sets of variables and arrays $I_{\max }$ which are different from an instance of ORDEREDDISTRIBUTE to another one (not the same number of variables and not the same restrictions on occurrences).

\section{Conclusion}

In this paper we presented a new global constraint, ORDEREDDistribute, which solves a practical modelling issue with respect to problems involving cost variables with strongly ordered domains. This constraint is complementary to global constraints based on statistics, such as Spread or Deviation. OrderedDistribute addresses those problems where variables can be carved in disjoint subsets, to control in a very precise way the number of occurrences of cost values within each subset. We provided a linear GAC filtering algorithm for ORDEREDDISTRIBUTE. We experimented successfully our global constraint on a cumulative problem with over-loads. 


\section{References}

1. A. Aggoun and N. Beldiceanu. Extending CHIP in order to solve complex scheduling and placement problems. Mathl. Comput. Modelling, 17(7):57-73, 1993.

2. F. Boussemart, F. Hemery, C. Lecoutre, and L. Saïs. Boosting systematic search by weighting constraints. Proc. ECAI, pages 146-150, 2004.

3. C. Mullinax and M. Lawley. Assigning patients to nurses in neonatal intensive care. Journal of the Operations Research Society, 53:25-35, 2002.

4. G. Pesant and J.-C. Régin. Spread: A balancing constraint based on statistics. Proc. CP, pages 460-474, 2005.

5. T. Petit and E. Poder. Global propagation of side constraints for solving overconstrained problems. To appear in the Annals of Operations Research, 2010.

6. T. Petit and J-C. Régin. The ordered global cardinality constraint. Research report 09-07-INFO, École des Mines de Nantes, 2009.

7. C.-G. Quimper. Enforcing domain consistency on the extended global cardinality constraint is np-hard. Technical Report CS-2003-39, School of Computer Science, University of Waterloo, 2003.

8. C.-G. Quimper, A. López-Ortiz, P. van Beek, and A. Golynski. Improved algorithms for the global cardinality constraint. Proc. CP, 2004.

9. J-C. Régin. Generalized arc consistency for global cardinality constraint. Proc. AAAI, pages 209-215, 1996.

10. J.-C. Régin and C. Gomes. The cardinality matrix constraint. Proc. CP, pages 572$587,2004$.

11. P. Schaus, Y. Deville, P. Dupont, and J-C. Régin. The deviation constraint. Proc. CPAIOR, 4510:260-274, 2007.

12. P. Schaus, P. Van Hentenryck, and J-C. Régin. Scalable load balancing in nurse to patient assignment problems. Proc. CPAIOR, 5547:248-262, 2009. 